\title{
CRYOGENICS FOR THE LARGE HADRON COLLIDER
}

\author{
$\mathrm{Ph}$. Lebrun
}

\begin{abstract}
The Large Hadron Collider (LHC), a $26.7 \mathrm{~km}$ circumference superconducting accelerator equipped with high-field magnets operating in superfluid helium below $1.9 \mathrm{~K}$, has now fully entered construction at CERN, the European Laboratory for Particle Physics. The heart of the LHC cryogenic system is the quasi-isothermal magnet cooling scheme, in which flowing two-phase saturated superfluid helium removes the heat load from the 36,000 ton cold mass, immersed in some $400 \mathrm{~m} 3$ static pressurised superfluid helium. The LHC also makes use of supercritical helium for non-isothermal cooling of the beam screens which intercept most of the dynamic heat loads at higher temperature. Although not used in normal operation, liquid nitrogen will provide the source of refrigeration for precooling the machine. Refrigeration for the LHC is produced in eight large refrigerators, each with an equivalent capacity of about $18 \mathrm{~kW}$ at $4.5 \mathrm{~K}$, completed by $1.8 \mathrm{~K}$ refrigeration units making use of several stages of hydrodynamic cold compressors. The cryogenic fluids are distributed to the cryomagnet strings by a compound cryogenic distribution line circling the tunnel. Procurement contracts for the major components of the LHC cryogenic system have been adjudicated to industry, and their progress will be briefly reported. Besides construction proper, the study and development of cryogenics for the LHC has resulted in salient advances in several fields of cryogenic engineering, which we shall also review.
\end{abstract}

LHC Division

Presented at the 16th International Conference on Magnetic Technology

26 September-2 October 1999 - Ponte Vedra Beach, USA

\footnotetext{
Administrative Secretariat

LHC Division

CERN

CH - 1211 Geneva 23

Switzerland 


\title{
Cryogenics for the Large Hadron Collider
}

\author{
Philippe Lebrun \\ CERN, European Organization for Nuclear Research, LHC Division, CH-1211 Geneva 23, Switzerland
}

\begin{abstract}
The Large Hadron Collider (LHC), a $26.7 \mathrm{~km}$ circumference superconducting accelerator equipped with highfield magnets operating in superfluid helium below $1.9 \mathrm{~K}$, has now fully entered construction at CERN, the European Laboratory for Particle Physics. The heart of the LHC cryogenic system is the quasi-isothermal magnet cooling scheme, in which flowing twophase saturated superfluid helium removes the heat load from the $36 ' 000$ ton cold mass, immersed in some $400 \mathrm{~m}^{3}$ static pressurised superfluid helium. The LHC also makes use of supercritical helium for non-isothermal cooling of the beam screens which intercept most of the dynamic heat loads at higher temperature. Although not used in normal operation, liquid nitrogen will provide the source of refrigeration for precooling the machine. Refrigeration for the LHC is produced in eight large refrigerators, each with an equivalent capacity of about $18 \mathrm{~kW}$ at 4.5 K, completed by $1.8 \mathrm{~K}$ refrigeration units making use of several stages of hydrodynamic cold compressors. The cryogenic fluids are distributed to the cryomagnet strings by a compound cryogenic distribution line circling the tunnel. Procurement contracts for the major components of the LHC cryogenic system have been adjudicated to industry, and their progress will be briefly reported. Besides construction proper, the study and development of cryogenics for the LHC has resulted in salient advances in several fields of cryogenic engineering, which we shall also review.
\end{abstract}

\section{INTRODUCTION}

The Large Hadron Collider (LHC), presently under construction at CERN, will be upon its completion in 2005 the largest scientific instrument in the world [1]. It will accelerate and bring into collision intense beams of protons and ions, in order to study the structure of matter and basic forces of nature on an unprecedentedly fine scale. To achieve this goal at an economically acceptable cost, the LHC is based on the generalised use of high-field superconducting magnets with $\mathrm{Nb}$ - Ti windings operating in superfluid helium at $1.9 \mathrm{~K}$. In CERN's tradition of maximising the scientific return of capital investment, the LHC will be installed in the existing $26.7-\mathrm{km}$ circumference tunnel which presently houses the LEP electronpositron collider, and will re-use the four large-capacity helium refrigerators of LEP [2], as well as the well-proven, smoothrunning network of pre-accelerators and injectors. The project also draws on several decades of know-how of the laboratory in the key technologies of applied superconductivity, cryogenics and ultra-high vacuum, not to mention the more classical sciences of accelerator engineering.
Besides its sheer size, the LHC also presents a number of challenges in accelerator physics and technology [3], which have demanded to conduct specific development programs over the last decade in several domains, among which highfield superconducting magnets [4] and helium cryogenics [5], in collaboration with national laboratories and industry in Europe, the U.S.A. and Japan. As concerns the cryogenics proper, these developments are now mainly completed, and the project has clearly entered its construction phase, with the ongoing industrial procurement of the main technical components. It is therefore appropriate at this stage to recall the main requirements and design constraints of the cryogenic system for the LHC, to describe its layout and main features, and to review its major contributions to progress in the field.

\section{DUTIES, CONSTRAINTS AND MAIN TECHNICAL CHOICES}

The main technical parameters of the LHC with proton beams, relevant to the design of the cryogenic system, are listed in Table 1.

Table 1 Main technical parameters of the LHC with proton beams

\begin{tabular}{lll}
\hline Circumference & 26.7 & $\mathrm{~km}$ \\
Beam energy in collision & 7 & $\mathrm{TeV}$ \\
Beam energy at injection & 0.45 & $\mathrm{TeV}$ \\
Bending field & 8.3 & $\mathrm{~T}$ \\
Coil aperture & 56 & $\mathrm{~mm}$ \\
Luminosity & $10^{34}$ & $\mathrm{~cm}^{-2} \cdot \mathrm{s}^{-1}$ \\
Beam intensity & 0.56 & $\mathrm{~A}$ \\
Beam stored energy & 350 & $\mathrm{MJ}$ \\
Radiated power per beam & 3.8 & $\mathrm{~kW}$ \\
Critical radiated photon energy & 44.1 & $\mathrm{eV}$ \\
Operating temperature & 1.9 & $\mathrm{~K}$ \\
Cold mass & $36 \times 10^{6}$ & $\mathrm{~kg}$ \\
\hline
\end{tabular}

The $7 \mathrm{TeV}$ beam energy in collision (14 TeV centre-ofmass) requires - over most of the circumference of the existing tunnel - a nominal bending field of $8.3 \mathrm{~T}$, i.e. a design field of about $9 \mathrm{~T}$ for the 1232, 15-m long twin-aperture superconducting dipole magnets. Using the well-established $\mathrm{Nb}-\mathrm{Ti}$ conductor technology, this may only be obtained by operating in pressurised superfluid helium below $2 \mathrm{~K}$, a solution pioneered at CEA, France and successfully implemented in the Tore Supra tokamak at the time of the first LHC studies [6]. However, as the specific heat of the superconducting alloy and its copper matrix fall rapidly with decreasing temperature, the full benefit of lower-temperature operation may only be reaped, in terms of stability margin, by making effective use of the particular transport properties of superfluid helium. The low bulk viscosity enables the coolant 
to permeate the heart of the magnet windings, while the very large specific heat (typically $10^{5}$ times that of the conductor per unit mass, $2 \times 10^{3}$ per unit volume), combined with the enormous heat conductivity at moderate flux $\left(10^{3}\right.$ times that of cryogenic-grade OFHC copper, peaking at $1.9 \mathrm{~K}$ ) may have a powerful stabilising action against thermal disturbances. To achieve this, the electrical insulation of the conductor must preserve sufficient porosity and thermal percolation paths while still fulfilling its demanding dielectric and mechanical duties.

A main difference with cooling a single, large magnet such as the toroid of a tokamak, however, stems from the geometrical configuration of large high-energy accelerators. The LHC magnets lie deep underground in a quasi-circular tunnel, composed of eight $3.3 \mathrm{~km}$-long sectors with access shafts to ground level and technical service areas only at the ends of each sector. Moreover, as CERN is located in suburban and rural areas between the city of Geneva and the piedmont of the Jura mountains, implantation of large technical infrastructure must fulfil strict environmental constraints. For the cryogenic engineer, this requires producing the refrigeration power in a limited number of sites scattered around the circumference, distributing it over kilometre lengths in a narrow tunnel, and using it efficiently in the longest cryostat in the world, to be assembled in situ. A direct consequence of these siting constraints is the cryogenic layout of the machine (Figure 1), with five cryogenic "islands" where all refrigeration and ancillary equipment is concentrated, both at ground level (electrical substation, compressor hall, cryogen storage, cooling towers, cold-boxes) and underground (lower cold-boxes, $1.8 \mathrm{~K}$ refrigeration units, interconnecting lines, distribution valve boxes). Each cryogenic island then feeds one or two adjacent tunnel sectors, which requires distributing and recovering the cooling fluids over distances of $3.3 \mathrm{~km}$ underground. This is achieved by means of a compound cryogenic distribution line [7], which runs along the cryomagnet strings in the tunnel and feeds every $106.9 \mathrm{~m}$-long lattice cell in parallel (Figure 2). The benefits of this series/parallel arrangement and the details of the cooling scheme in the cryo-magnets proper will be presented in the following.

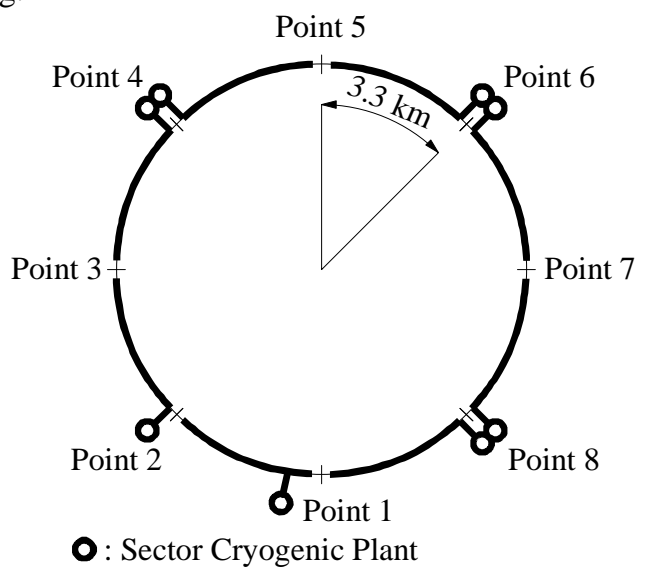

Figure 1 Overall layout of the LHC cryogenic system

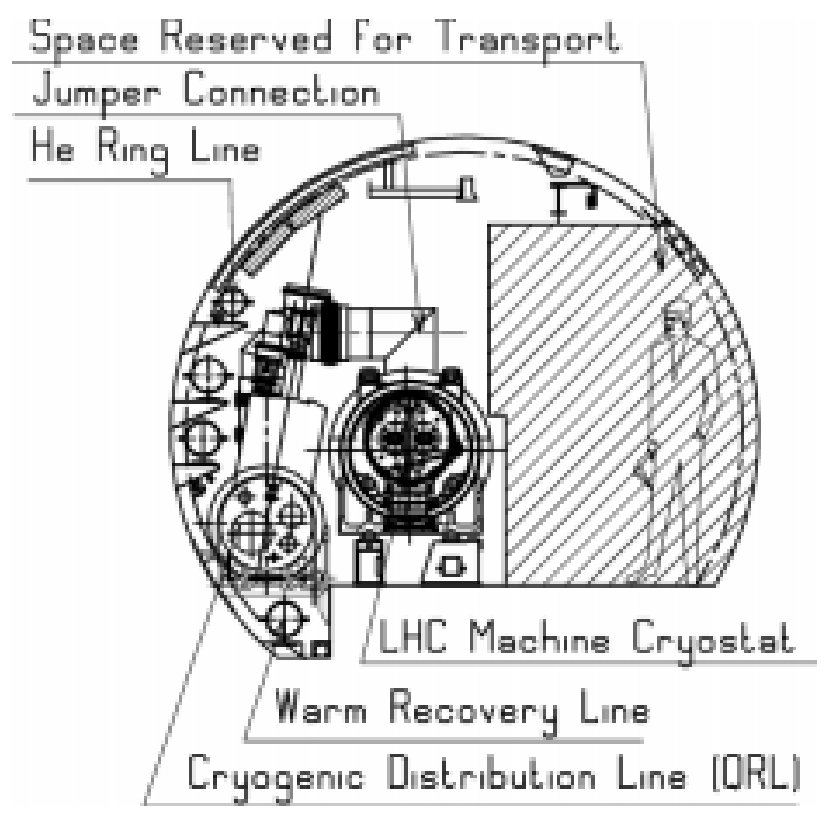

Figure 2 Transverse cross-section of the LHC tunnel

Besides the above geographic considerations, the tunnel lies for geo-technical reasons, in a plane with a slope of $1.4 \%$ with respect to the horizontal, thus resulting in elevation differences of up to $120 \mathrm{~m}$ across the tunnel diameter. Both aspects raised concern about hydrostatic heads and risk of flow instabilities for liquid-vapour systems or near-critical fluids such as often encountered in cryogenics.

The LHC cryogenic system is required to:

- maintain the pressurised superfluid helium bath of all cryo-magnets around the ring at their nominal operating temperature below $1.9 \mathrm{~K}$,

- cool down from ambient temperature and fill (respectively empty and warm up to ambient temperature) each sector of the machine in less than two weeks,

- accommodate resistive transitions of full cells of the machine lattice and recover from these transitions in less than two hours, while minimising loss of cryogen and system perturbations.

In addition to these basic operational duties, the LHC cryogenic system should allow for rapid cool-down and warmup of limited lengths of cryo-magnet strings, e.g. for repairing or exchanging a defective unit. It should also be able to cope with the resistive transition of a full-sector - this defining the maximum credible accident - without impairing personnel or equipment safety. Finally, to ensure reliable operation, it should provide some redundancy of functions among its components and sub-systems.

To fulfil these requirements within the above constraints and boundary conditions, the LHC cryogenic system makes use of helium in several thermodynamic states, shown in Figure 3 on a pressure-temperature phase diagram. The corresponding flow-scheme of a LHC lattice cell [8] appears in Figure 4. As already mentioned, the cryo-magnets operate in a static bath of pressurised helium II, cooled by heat exchange with flowing saturated helium II. 


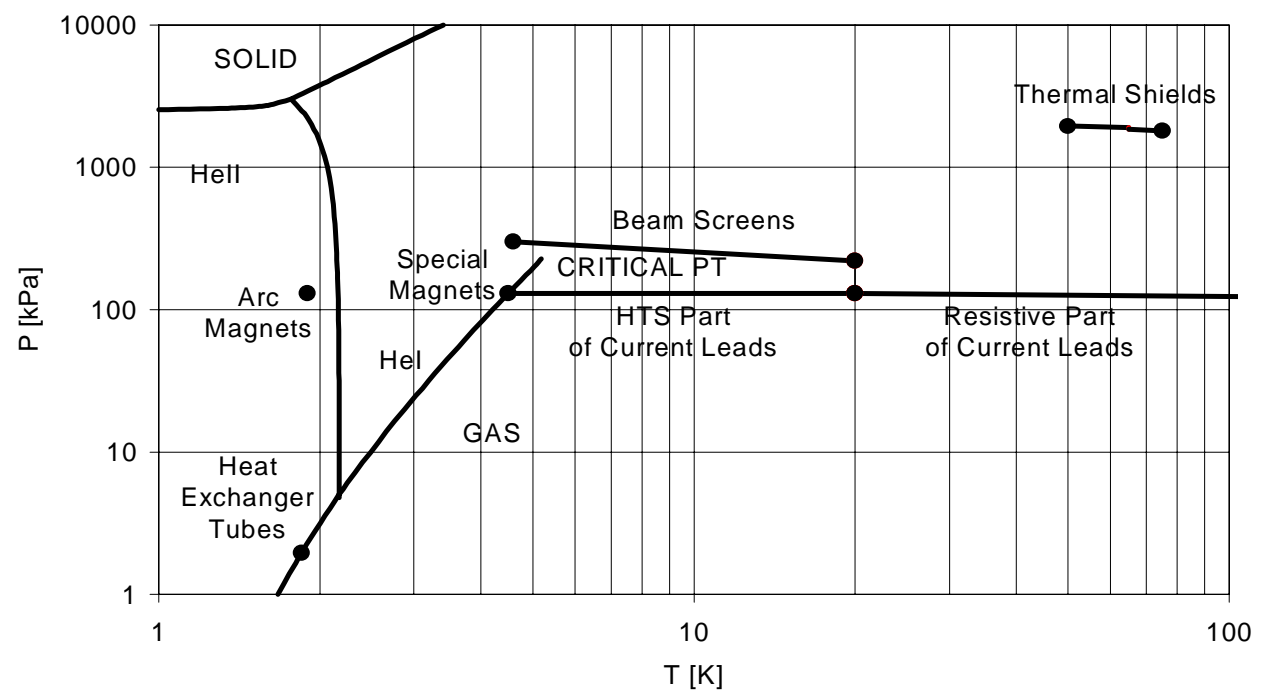

Figure 3 Thermodynamic states of helium in the LHC cryogenic system
Low-pressure vapour resulting from the vaporisation of the saturated helium II is returned to the refrigerator by header B. Supercritical helium, distributed by header C, is used to fill the cryo-magnet baths, to produce - after subcooling and JouleThomson expansion - the saturated helium II flowing in the heat exchanger tubes, and to feed line $\mathrm{C}^{\prime}$ in series with the beam screens operating between 5 and $20 \mathrm{~K}$. The resulting gaseous helium is returned to the refrigerator by line $\mathrm{D}$. The first level of thermal shielding and heat interception in the magnet cryostats and the distribution line is provided by forced circulation of gaseous helium under pressure at temperature between 50 and $75 \mathrm{~K}$, through lines $\mathrm{E}$ and $\mathrm{F}$ respectively. Special magnets which do not require cooling at $1.9 \mathrm{~K}$ operate in baths of saturated helium I at $4.5 \mathrm{~K}$. Such baths are also used to cool the lower part of HTS-based current leads, the upper, resistive part of which is cooled by gaseous helium between $20 \mathrm{~K}$ and ambient temperature. We shall return to the specific features, operating functions and cryogenic performance of these different circuits in the following chapters.

\section{SUPERFLUID-HELIUM MAGNET COOLING SCHEME}

The large, but finite bulk thermal conductivity of static pressurised superfluid helium has been successfully applied for cooling devices below $2 \mathrm{~K}$ and transporting their heat loads over distances of up to a few tens of metres. Cooling the kilometre-long strings of magnets in the LHC sectors within a narrow temperature range, say $0.1 \mathrm{~K}$, is however beyond the capability of helium-II thermal conduction alone [9]. In order to maintain below $1.9 \mathrm{~K}$ the furthest magnet of each sector, each 106.9 m-long LHC cell is constituted of a single bath of static pressurised superfluid helium, cooled from a quasiisothermal heat sink in the form of a bayonet heat exchanger running through the magnet string [10], and in which the latent heat of a stratified two-phase flow of saturated helium II absorbs the applied heat load, about $0.4 \mathrm{~W} / \mathrm{m}$ in the LHC arcs (Figure 4).
The validity and performance of such a scheme depend critically on the thermo-hydraulic behaviour of two-phase helium II flowing in quasi-horizontal tubes: this has been the subject of theoretical modelling and experimental studies at CERN [11] and CEA Grenoble (France) [12]. These studies have demonstrated that, over a large range of vapour quality, most of the tube cross-section is occupied by the vapour flow, which then controls the overall pressure drop. For vapour velocities of up to a few $\mathrm{m} / \mathrm{s}$, the drag between the two phases remains small, so that the liquid flows separated from the vapour, almost as in single phase and open channel. In this condition, the heat transfer is mainly controlled by the wetted area inside the tube, which can be adequately predicted by simple models for engineering purpose. Other important factors controlling the heat transfer across the tube wall are the conductivity of the tube material and the Kapitza thermal resistance at the solid-liquid interfaces [13]. By using a 53.4$\mathrm{mm}$ inner diameter tube of OFHC copper with a wall thickness of $2.3 \mathrm{~mm}$, the total transverse impedance when fully wetted can be kept down to about $0.3 \mathrm{mK} \cdot \mathrm{m}^{2} . \mathrm{W}^{-1}$, and the practical heat transfer capability of the partially wetted bayonet heat exchanger is thus typically a few mK.m. $\mathrm{W}^{-1}$. Based on this measured performance, the temperature profiles of the LHC sectors can be calculated with confidence (Figure 5), meeting the demanding requirement of $0.1 \mathrm{~K}$ between the furthest cryomagnet and the sector refrigerator. The final validation of this cooling scheme was performed on a 35-m long prototype magnet string which has accumulated about 13'000 hours operation at $1.9 \mathrm{~K}$, undergoing successfully all possible nominal and off-design operating conditions [14]. This quasifull scale working model of a LHC cell enabled to address dynamic control aspects, particularly demanding on this system characterised by strong non-linearity - including initial inverse response - and long time delays induced by the low velocity of the liquid flow. To cope with this, we have been investigating and have begun to implement advanced automation techniques [15], such as linear model-based predictive control [16], which may significantly improve the temperature regulation of the cryo-magnets. 


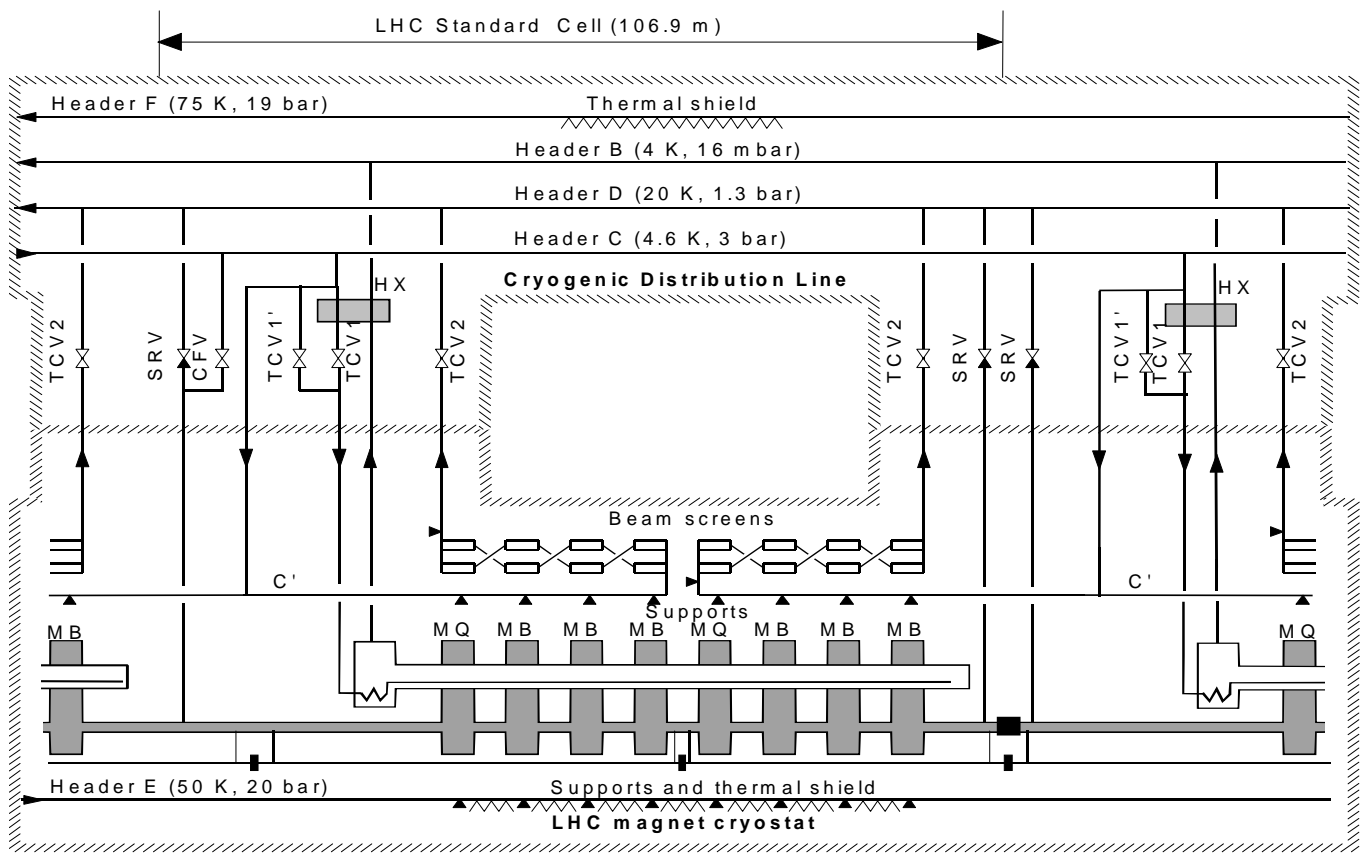

Figure 4 Cryogenic flowscheme of LHC lattice cell

To complete this chapter, it is worth mentioning that the lowbeta quadrupoles in the interaction regions of the LHC, subject to much higher heat loads in the $10 \mathrm{~W} / \mathrm{m}$ range, will be cooled by a similar scheme, using larger-diameter, corrugated heat exchanger tubes made of copper [17]. A full-scale experimental set-up modelling this cooling loop, designed and built by FNAL Batavia (U.S.A.), is due to begin operation at CERN by early 2000.

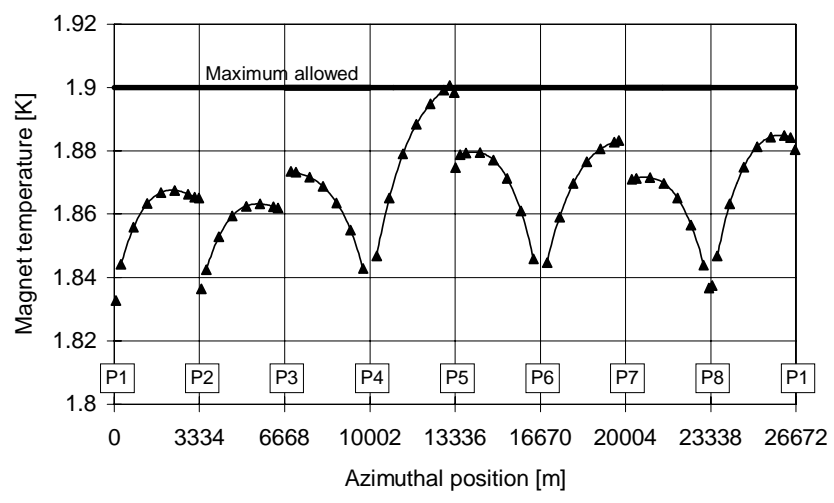

Figure 5 Calculated temperature profiles of LHC magnets in nominal operation

\section{THERMAL DESIGN AND INSULATION TECHNIQUES}

In view of the high thermodynamic cost of refrigeration at $1.8 \mathrm{~K}$, the thermal design of the LHC cryogenic components aims at intercepting the largest fraction of applied heat loads at higher temperature, hence the multiple, staged temperature levels in the system (Table 2).

The steady-state heat loads are of three basic types:

- heat inleaks, resulting from the design of the cryostats,

- resistive heating in the non-superconducting splices, which have to be taken by the superfluid helium bath,
- beam-induced loads, dominated by synchrotron radiation, dissipation of beam-image currents, and impingement of photo-electrons accelerated by the beam potential.

The latter are mostly intercepted by the beam screens equipping the magnet apertures, cooled by circulation of supercritical helium between 5 and $20 \mathrm{~K}$. In comparison with the above, the large transient heat loads produced by magnetic hysteresis and eddy current dissipation during current ramp and discharge can only be buffered by the heat capacity of the $15 \mathrm{l} / \mathrm{m}$ helium in the magnet cold mass.

Table 2 Distributed steady-state heat loads in an LHC arc [W/m]

\begin{tabular}{lcccc}
\hline Temperature & $50-75 \mathrm{~K}$ & $4.5-20 \mathrm{~K}$ & $4 \mathrm{~K}$ & $1.9 \mathrm{~K} \mathrm{LHe}$ \\
\hline Heat inleaks* & 6.7 & 0.27 & 0.05 & 0.20 \\
Resistive heating & $\varepsilon$ & 0.01 & 0 & 0.13 \\
Beam-induced & 0 & 0.91 & 0 & 0.07 \\
\hline Total & 6.7 & 1.19 & 0.05 & 0.40 \\
\hline * No contingency & & & &
\end{tabular}

The cryostats [18] and cryogenic distribution line [7] combine several low-temperature insulation and heat interception techniques which will have to be reliably implemented on an industrial scale (Figure 6). These include low-conduction support posts made of non-metallic glassfibre/epoxy composite [19], low-impedance thermal contacts under vacuum for heat intercepts and multi-layer reflective insulation wrapping the some $80^{\prime} 000 \mathrm{~m}^{2}$ of cold surface area below $20 \mathrm{~K}$ [20]. Each of these techniques was investigated and validated separately in the laboratory [21], and the complete cryostats were then modelled by thermal network analysis and optimised in standard as well as off-design operation [22]. Precision experimental measurements confirmed the soundness of the adopted design and its suitability for industrial construction [23]. 


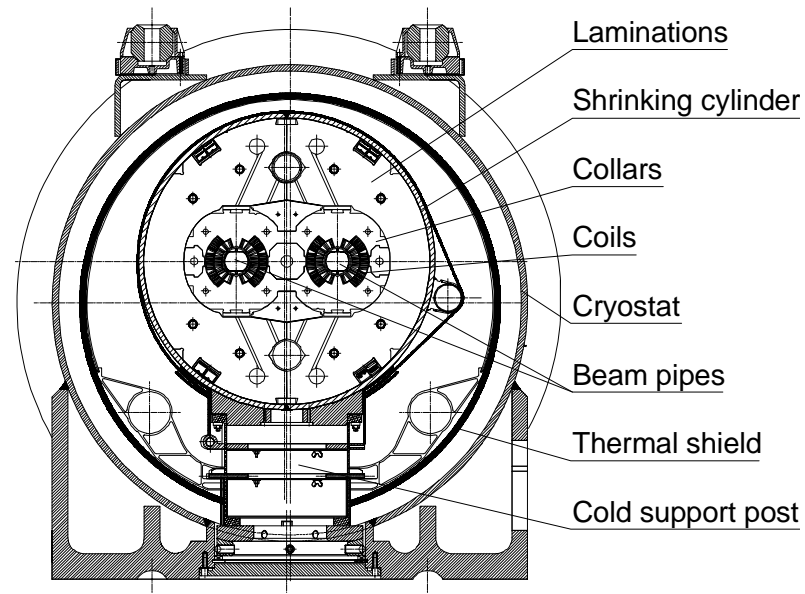

Figure 6 Transverse cross-section of LHC dipole cryo-magnet

\section{SUPERCRITICAL-HELIUM COOLING OF THE BEAM SCREENS}

Besides their primary function of intercepting beam-induced heat loads at a temperature well above that of the magnets, the beam screens also act as an intermediate-temperature baffle for the cryopump constituted by the $1.9 \mathrm{~K}$ surface of the magnet bores, thus sheltering the cold surface from synchrotron radiation, preventing desorption of the trapped gas molecules and avoiding breakdown of the beam vacuum [24]. In order to limit resistive heating as well as residual heat inleaks to the magnets, the beam screens must operate below about $30 \mathrm{~K}$. To match the available temperature levels in the existing refrigerators, they are cooled non-isothermally by a forced flow of weakly supercritical helium, between 4.6 and $20 \mathrm{~K}$, which reduces the entropic load by a factor 8 with respect to $1.9 \mathrm{~K}$ isothermal refrigeration. The choice of monophase, supercritical helium aims at avoiding the potential problems of two-phase flow in long, narrow channels. However, the strongly varying properties of helium close to the critical point may nevertheless create thermohydraulic instabilities, while the large aspect ratio of the cooling channels induces long control delays. These problems have been investigated both theoretically and experimentally on a full-scale test loop [25], thus permitting to identify critical parameters and to validate the steady-state and transient performance of the proposed solution [26].

\section{LARGE-CAPACITY REFRIGERATION AT 4.5 K AND $1.8 \mathrm{~K}$}

The refrigeration demands of the LHC sectors [27], listed in Table 3 which also includes contingency for excess capacity and uncertainty in heat load budgets, require mixed-duty operation of the cryogenic helium refrigerators, in order to fulfil a variety of isothermal and non-isothermal cooling duties. This amounts to a total equivalent entropic capacity of $120 \mathrm{~kW} / 4.5 \mathrm{~K}$, thus making the LHC the world's most powerful helium refrigeration system.

Previous experience at CERN with large cryogenic helium plants delivered by European industry in the framework of turn-key contracts, has demonstrated their dependable performance, good efficiency and high operational reliability
[28]. Consequently, CERN has issued in 1997 a functional and interface specification for the procurement of four such new refrigerators to equip the "high-load" sectors [29], in complement to the existing LEP units which have been upgraded in capacity and will feed the "low-load" sectors of the LHC. The adjudication rule took into consideration, besides capital investment, the integrated costs of operation over a period of ten years, thus giving a premium to efficiency, and indirectly - compactness [30]. On the basis of the offers received, contracts were adjudicated to Air Liquide (France) and Linde Kryotechnik (Switzerland) for the supply of two refrigerators each. Although based on different variants of the Claude cycle, both types of machines are designed to reach similar efficiencies, around $30 \%$ with respect to the Carnot cycle [31, 32]. The first of these four refrigerators is due to be commissioned in late 2000.

Table 3 Installed refrigeration capacity in the LHC sectors

\begin{tabular}{lccc}
\hline $\begin{array}{c}\text { Temperature } \\
\text { level }\end{array}$ & High-load sector & Low-load sector & \\
\hline $50-75 \mathrm{~K}$ & 33000 & 31000 & {$[\mathrm{~W}]$} \\
$4.6-20 \mathrm{~K}$ & 7700 & 7600 & {$[\mathrm{~W}]$} \\
$4.5 \mathrm{~K}$ & 300 & 150 & {$[\mathrm{~W}]$} \\
$1.8 \mathrm{~K}$ & 2400 & 2100 & {$[\mathrm{~W}]$} \\
$3-4 \mathrm{~K}$ & 430 & 380 & {$[\mathrm{~W}]$} \\
$20-280 \mathrm{~K}$ & 41 & 27 & {$[\mathrm{~g} / \mathrm{s}]$} \\
\hline
\end{tabular}

The efficient production of $1.8 \mathrm{~K}$ refrigeration in the $\mathrm{kW}$ range - a novel requirement set by the LHC project - may only be achieved practically through combined cycles making use of sub-atmospheric cryogenic compressors and heat exchangers [33]. To foster the development of these technologies, CERN has procured from industry prototype low-pressure heat exchangers of different designs, presently under evaluation at CEA, Grenoble (France), as well as three hydrodynamic compressors, each handling $18 \mathrm{~g} / \mathrm{s}$ at $1 \mathrm{kPa}$ suction pressure, with a pressure ratio of 3 [34]. The thorough test campaigns performed on the latter have permitted to investigate such critical issues as impeller and diffuser hydrodynamics, mechanical and thermal design, drive and bearing technology, as well as their impact on overall efficiency. Design and optimisation studies have also been performed, in liaison with industry, on refrigeration cycles meeting the requirements and boundary conditions of the project, and matched to the expected performance of full-size machinery [35]. This preparatory work has permitted to launch in 1998 the industrial procurement of the eight $1.8 \mathrm{~K}$ refrigeration units for the LHC sectors, through a successful call for tenders based on a functional and interface specification. Contracts have been adjudicated to Air Liquide (France) and a consortium of IHI (Japan) and Linde Kryotechnik (Switzerland) for the delivery and installation of four units each. The overall coefficient-ofperformance of these $1.8 \mathrm{~K}$ refrigeration units, once attached to the main $4.5 \mathrm{~K}$ refrigerators of the $\mathrm{LHC}$, is expected to be around $900 \mathrm{~W} / \mathrm{W}$. 


\section{CURRENT LEADS USING HIGH-TEMPERATURE SUPERCONDUCTORS}

Powering the different magnet circuits of the LHC will require feeding about 3.6 MA into the cryogenic environment, which would result in a heavy liquefaction burden when using conventional resistive vapour-cooled leads. The advent of quasi-industrial HTS materials, combined with the favourable cooling conditions provided by the $20 \mathrm{~K}$ level in the LHC cryogenic system [36], renders the use of HTS-based current leads very attractive, as it allows higher design current densities than usually permitted by liquid nitrogen cooling at $80 \mathrm{~K}$ [37]. As compared to their conventional counterparts, the HTS-based leads of the LHC have the potential of reducing the entropic load by a factor of 3 , thus saving the equivalent capacity of a ninth sector refrigerator. After conducting tests on material samples, CERN has procured from industry and is currently testing in a dedicated set-up [38], prototypes of such leads for $13 \mathrm{kA}$ and $0.6 \mathrm{kA}$, based on several alternative choices of material and manufacturing technique [39]. Upon completion of this programme in 2000, the final specifications for the LHC current leads will be established, and the procurement of the series units undertaken.

\section{COPING SAFELY WITH MAGNET RESISTIVE TRANSITIONS}

In the first tenths of a second following the transition of a dipole magnet, the $500 \mathrm{~kJ} / \mathrm{m}$ stored magnetic energy will be dissipated in the resistive windings, and part of it eventually released, on a much longer time scale, to the static helium in the cold mass. To contain the resulting pressure rise within the $2 \mathrm{MPa}$ design pressure of the helium enclosure, helium will have to be discharged at high flow-rate into header D, normally kept cold by the $20 \mathrm{~K}$ bleed from the beam-screen cooling circuits. The large acceptance of this header enables it to perform as a temporary buffer storage, thus avoiding the need for further helium discharge in case of resistive transitions affecting limited stretches of magnets (one to a few lattice cells) [40]. For generalised resistive transitions, cold gaseous helium will be discharged and recovered, though at much lower flow-rates, from header D into the $2 \mathrm{MPa}$ gas storage vessels at ambient temperature, located in the service areas at ground surface around the LHC circumference [41].

The discharge of helium from the magnet cold mass will be performed, every $106.9 \mathrm{~m}$, by cold safety relief valves, normally closed with their inlet under pressurised helium II. Such components, which are not available off-the-shelf, had to be developed by industry following CERN specification [42], and their performance assessed on a specially designed test facility [43]. The completion of the prototype development programme in 2000 will permit to undertake the procurement of the some 400 series units for the LHC ring.

The thermohydraulic consequences of resistive transitions in the cryo-magnets may constitute a significant, but not exclusive source of risk to personnel, or equipment. A systematic assessment of such risks in the LHC cryogenic system, based on a tentatively exhaustive analysis of the occurrence of accidental events and the gravity of their consequences, has been recently conducted [44]. Specific design features of the system, such as the large acceptance of the cold recovery header $\mathrm{D}$, the absence of liquid nitrogen underground and of helium pumps in the cooling loops, strongly contribute to intrinsic safety.

\section{PRECISION THERMOMETRY BELOW $4 \mathrm{~K}$}

The tight temperature margins allowed along the cryomagnet strings in the LHC require to implement precision cryogenic thermometry (overall measurement uncertainty down to $\pm 10 \mathrm{mK}$ ) on an industrial scale (several thousand channels) with long-term robustness and reliability. Following the construction and commissioning of cryogenic calibration facilities of metrological class at CERN [45] and CNRS Orsay (France) [46], several types of sensors have been tested on statistically significant ensembles, as regards their performance in LHC environmental conditions. In particular, the effects of neutron irradiation at cryogenic temperature [47] and of thermal cycling [48] have been investigated on several hundred thermometers, in view of selecting adequate solutions for the project. The calibration of the series sensors will be performed by CNRS Orsay (France).

The stringent requirements on temperature measurements, once applied to signal conditioning, cannot be met by commercially available equipment. Several conditioner architectures which could simultaneously provide the large dynamic range, accuracy, stability and tolerance to radiation levels such as encountered in the LHC, have been investigated and prototyped in discrete component as well as ASIC versions [49].

\section{CONClusion}

After some ten years of pluridisciplinary $R \& D$, the novel technical choices for the LHC cryogenic system have been validated, and are now being implemented as project construction is progressing and industrial procurement of the series components and sub-systems is under way.

\section{ACKNOWLEDGEMENTS}

The work reported here is that of my colleagues in the LHC Accelerator Cryogenics group. The help of L. Tavian in preparing this paper is gratefully acknowledged.

\section{REFERENCES}

[1] L. Evans, The Large Hadron Collider: present status and prospects, invited paper at this conference.

[2] S. Claudet, W. Erdt, P. Frandsen, Ph. Gayet, N. Solheim \& G. Winkler, Four $12 \mathrm{~kW} / 4.5 \mathrm{~K}$ cryoplants at CERN, Cryogenics 34 ICEC Supplement (1994) 99-102.

[3] L. Evans, LHC accelerator physics and technology challenges, invited paper at PAC'99, New York (1999).

[4] K. Artoos et al., Design, manufacturing status, first results of the LHC main dipole final prototypes, and steps towards series manufacture, paper presented at this conference.

[5] Ph. Lebrun, Advances in cryogenics at the Large Hadron Collider invited paper at ICEC17, Bournemouth (1998). 
[6] G. Claudet \& R. Aymar, Tore Supra and helium-II cooling of large high-field magnets, Adv. Cryo. Eng. 35A (1990) 55-67.

[7] W. Erdt, G. Riddone \& R. Trant, The cryogenic distribution line for the LHC: functional specification and conceptual design, paper presented at CEC'99 Montreal (1999).

[8] M. Chorowski, W. Erdt, Ph. Lebrun, G. Riddone, L. Serio, L Tavian, U. Wagner and R. van Weelderen, A simplified cryogenic distribution scheme for the Large Hadron Collider, Adv. Cryo. Eng. 43A (1998) 395-402.

[9] Ph. Lebrun, Superfluid helium as a technical coolant, Atti XV Congresso Nazionale sulla Trasmissione del Calore, Edizioni ETS, Politecnico di Torino, Italy (1997) 61-77.

[10] Ph. Lebrun, L. Serio, L. Tavian \& R. van Weelderen, Cooling strings of superconducting devices below $2 \mathrm{~K}$ : the helium II bayonet heat exchanger, Adv. Cryo. Eng. 43A (1998) 419-426.

[11] J. Casas, A. Cyvoct, Ph. Lebrun, M. Marquet, L. Tavian \& R. van Weelderen, Design concept and first experimental validation of the superfluid helium system for the Large Hadron Collider, Cryogenics 32 ICEC Supplement (1992) 118-121.

[12] A. Gauthier, L. Grimaud, B. Rousset, A. Bézaguet \& R. van Weelderen, Thermohydraulic behaviour of $\mathrm{He}$ II in stratified cocurrent two-phase flow, Proc. ICEC16, Elsevier Science, Oxford, UK (1997) 519-522.

[13] D. Camacho, S. Chevassus, C. Policella, J.M. Rieubland, G. Vandoni \& R. van Weelderen, Thermal characterisation of the LHC HeII heat exchanger tube, Proc. ICEC17, IoP, Bristol, UK (1998) 647-650.

[14] J. Casas-Cubillos, Ph. Provenaz, L. Serio, A. Suraci \& R. van Weelderen, Operation, testing and long-term behaviour of the LHC Test String cryogenic system, Proc. ICEC17, IoP, Bristol, UK (1998) 747-750.

[15] B. Flemsaeter, E. Blanco, J. Casas-Cubillos, C. de Prada \& S Saelid, Applying advanced control techniques for the temperature regulation of the LHC superconducting magnets, Proc. ICEC17, IoP, Bristol, UK (1998) 631-634.

[16] E. Blanco-Viñuela, J. Casas-Cubillos \& C. de Prada-Moraga, Linear model-based predictive control of the LHC $1.8 \mathrm{~K}$ cryogenic loop, paper presented at CEC'99 Montreal (1999).

[17] R. Byrns, Y. Huang, J. Kerby, Ph. Lebrun, L. Morrison, T. Nicol, T. Peterson, R. Trant, R. van Weelderen \& J. Zbasnik, The cryogenics of the LHC interaction-region final-focus superconducting magnets, Proc. ICEC17, IoP, Bristol, UK (1998) 743-746.

[18] J.C. Brunet, V. Parma, G. Peon, A. Poncet, P. Rohmig, B. Skoczen $\&$ L. Williams, Design of the second-series of LHC prototype dipole magnet cryostats, Adv. Cryo. Eng. 43A (1998) 435-441.

[19] M. Mathieu, V. Parma, T. Renaglia, P. Rohmig \& L. Williams, Supporting systems from $293 \mathrm{~K}$ to $1.9 \mathrm{~K}$ for the LHC cryomagnets, Adv. Cryo. Eng. 43A (1998) 427-434.

[20] G. Ferlin, B. Jenninger, Ph. Lebrun, G. Peon, G. Riddone \& B Szeless, Comparison of floating and thermalised multilayer insulation systems at low boundary temperature, Proc. ICEC16, Elsevier Science, Oxford, UK (1997) 443-446.

[21] D. Camacho, S. Chevassus, G. Ferlin, M. Pangallo, C. Policella J.M. Rieubland, L. Simon \& G. Vandoni, Heat inleak measurement on LHC components, paper presented at CEC'99 Montreal (1999).

[22] G. Riddone, Analisi teorica e verifica sperimentale delle prestazioni termiche dei criostati prototipi del progetto LHC, Doctoral thesis, Politecnico di Torino, Italy (1997).

[23] V. Benda, L. Dufay, G. Ferlin, Ph. Lebrun, J.M. Rieubland, G. Riddone, B. Szeless, L. Tavian \& L. Williams, Measurement and analysis of thermal performance of LHC prototype cryostats, $\underline{\mathrm{Adv}}$. Cryo. Eng. 41A (1996) 785-792.

[24] O. Gröbner, The LHC vacuum system, Proc. PAC'97 3, IEEE Piscataway, NJ, USA (1998) 3542-3546.

[25] E. Hatchadourian, Ph. Lebrun \& L. Tavian, Supercritical helium cooling of the LHC beam screens, Proc. ICEC17, IoP, Bristol, UK (1998) 793-796.

[26] E. Hatchadourian, Stability and control of supercritical helium flow in the LHC circuits, paper presented at CEC'99 Montreal (1999).

[27] Ph. Lebrun, G. Riddone, L. Tavian \& U. Wagner, Demands in refrigeration capacity for the Large Hadron Collider, Proc. ICEC16, Elsevier Science, Oxford, UK (1997) 95-98.

[28] M. Barranco-Luque, S. Claudet, W. Erdt, P. Frandsen, Ph. Gayet, D. Güsewell, Ph. Lebrun, J. Schmid, N. Solheim, Ch. Titcomb, U.
Wagner \& G. Winkler, Conclusions from procuring, installing and commissioning six large-scale helium refrigerators at CERN, Adv. Cryo. Eng. 41A (1996) 761-768.

[29] S. Claudet, Ph. Gayet \& U. Wagner, Specification of four new large $4.5 \mathrm{~K}$ refrigerators for the $\mathrm{LHC}$, paper presented at CEC'99 Montreal (1999).

[30] S. Claudet, Ph. Gayet, Ph. Lebrun, L. Tavian \& U. Wagner, Economics of large helium cryogenic systems: experience from recent projects at CERN, paper presented at CEC'99 Montreal (1999)

[31] P. Dauguet, G.M. Gistau-Baguer \& P. Briend, Two large $18 \mathrm{~kW}$ (equivalent power at $4.5 \mathrm{~K}$ ) helium refrigerators for CERN's LHC project, supplied by Air Liquide, paper presented at CEC'99 Montreal (1999).

[32] J. Bösel, B. Chromec \& A. Meier, Two large $18 \mathrm{~kW}$ (equivalent power at $4.5 \mathrm{~K}$ ) helium refrigerators for CERN's LHC project, supplied by Linde Kryotechnik AG, paper presented at CEC'99 Montreal (1999).

[33] Ph. Lebrun, L. Tavian \& G. Claudet, Development of large-capacity refrigeration at $1.8 \mathrm{~K}$ for the Large Hadron Collider, Proc. Kryogenika'96, Icaris, Praha, Czech Republic (1996) 54-59.

[34] A. Bézaguet, Ph. Lebrun \& L. Tavian, Performance assessment of industrial prototype cryogenic helium compressors for the Large Hadron Collider, Proc. ICEC17, IoP, Bristol, UK (1998) 145-148.

[35] F. Millet, P. Roussel, L. Tavian \& U. Wagner, A possible $1.8 \mathrm{~K}$ refrigeration cycle for the Large Hadron Collider, Adv. Cryo. Eng. 43A (1998) 387-393.

[36] A. Ballarino, A. Ijspeert \& U. Wagner, Potential of hightemperature superconductor current leads for LHC cryogenics, Proc. ICEC16, Elsevier Science, Oxford, UK (1997) 1139-1142.

[37] A. Ballarino, Discendenti di corrente realizzati con materiale superconduttore ad alta temperatura critica per l'alimentazione dei magneti del Large Hadron Collider, Doctoral thesis, Politecnico di Torino, Italy (1998).

[38] A. Ballarino, A. Bézaguet, P. Gomes, L. Métral, L. Serio \& A. Suraci, A low heat inleak cryogenic station for testing HTS current leads for the Large Hadron Collider, paper presented at CEC'99 Montreal (1999).

[39] A. Ballarino, High-temperature superconducting current leads for the Large Hadron Collider, Proc. IEEE 9 (1999) 523-526.

[40] M. Chorowski, Ph. Lebrun, L. Serio \& R. van Weelderen, Thermohydraulics of quenches and helium recovery in the LHC prototype magnet strings, Cryogenics 38 (1998) 533-543.

[41] M. Chorowski \& B. Skoczen, Thermo-mechanical analysis of cold helium injection into gas storage tanks made of carbon steel following resistive transitions of the LHC magnets, Proc. ICEC17, IoP, Bristol, UK (1998) 755-758.

[42] L. Dufay, A. Perin \& R. van Weelderen, Characterisation of prototype superfluid helium safety relief valves for the LHC magnets, paper presented at CEC'99 Montreal (1999).

[43] A. Bézaguet, L. Dufay, G. Ferlin, R. Losserand-Madoux, A. Perin, G. Vandoni \& R. van Weelderen, A facility for accurate heat load and mass leak measurements on superfluid helium valves, paper presented at CEC'99 Montreal (1999).

[44] M. Chorowski, Ph. Lebrun \& G. Riddone, Preliminary risk analysis of the LHC cryogenic system, paper presented at CEC'99 Montreal (1999).

[45] C. Balle, J. Casas \& J.P. Thermeau, Cryogenic calibration facility at CERN, Adv. Cryo. Eng. 43A (1998) 741-748.

[46] E. Chanzy, T. Junquera, J.P. Thermeau, S. Buhler, Ch. Joly, J. Casas-Cubillos \& Ch. Balle, Cryogenic thermometer calibration system using a helium cooling loop and a temperature controller, Proc. ICEC17, IoP, Bristol, UK (1998) 751-754.

[47] J.F. Amand, J. Casas-Cubillos, T. Junquera \& J.P. Thermeau, Neutron irradiation tests in superfluid helium of LHC cryogenic thermometers, Proc. ICEC17, IoP, Bristol, UK (1998) 727-730.

[48] Ch. Balle, J. Casas-Cubillos, J.M. Rieubland, A. Suraci, F. Togny \& N. Vauthier, Influence of thermal cycling on cryogenic thermometers, paper presented at CEC'99 Montreal (1999).

J. Casas, P. Gomes, K.N. Henrichsen, U. Jordung \& M.A. Rodriguez-Ruiz, Signal conditioning for cryogenic thermometry in the LHC, paper presented at CEC'99 Montreal (1999). 